\title{
The therapeutic value of cerebrospinal fluid ctDNA detection by next-generation sequencing for meningeal carcinomatosis: a case report
}

\author{
Xiaosu Guo, Junzhao Cui, Yue Zhao, Weixin Han, Yueli Zou, Ruiping Gao, Qing Li, Xiaoqing Li, Junying He \\ and Hui Bu* (D)
}

\begin{abstract}
Background: It is usually very complicated to treat meningeal carcinomatosis, and it is important to treat it as soon as possible.

Case presentation: The 19-Del mutation was found in the exon for the epidermal growth factor receptor gene in the pleural effusion of a patient on March 11th, 2015. He took $250 \mathrm{mg}$ of oral gefitinib once a day for 11 months beginning in December of 2015. On the 3rd of November 2016, he arrived at the hospital and presented with dizziness, headache and transient blurred vision. At this time, he began to take $4 \mathrm{mg}$ of oral zoledronic acid once a month to prevent bone metastases. The result of a cytology exam of the cerebrospinal fluid showed that the man had meningeal carcinomatosis. The 19-Del mutation and the 20-T790 M mutation in the exon of the epidermal growth factor receptor gene was found by the next generation sequencing of the CSF. Then, he discontinued taking gefitinib and began to take 90-100 mg of oral AZD9291 once a day in November 2016. After adjusting the medication dose based on the NGS, his headache was noticeably reduced, and his condition gradually stabilized.
\end{abstract}

Conclusions: Cerebrospinal fluid ctDNA detection by next generation sequencing may become a suitable biomarker to monitor clinical treatment response in meningeal carcinomatosis.

Keywords: Meningeal carcinomatosis, Cerebrospinal fluid, ctDNA, Next generation sequencing, Drug treatment

\section{Background}

Meningeal carcinomatosis (MC) is a rare complication of extraneural solid tumors and consists of a focal or multiple malignant infiltration of neoplastic cells in the leptomeninges of the brain or spinal cord [1]. It is well recognized that $\mathrm{MC}$ occurs in $1-5 \%$ of patients with solid tumors. It is reported that $5 \%$ of patients with breast cancer, $23 \%$ of patients with melanoma and 9$25 \%$ of patients with small-cell lung cancer can develop MC [1]. Some clinical symptoms of MC include headache and nonspecific nerve palsy, and the patient's condition can deteriorate quite quickly. In addition, $\mathrm{MC}$ is usually fatal to patients with advanced cancer with a

\footnotetext{
* Correspondence: buhui881@163.com

Department of Neurology, The Second Hospital of Hebei Medical University, No 215, Peace Road, Shijiazhuang, China
}

mean survival time of 4 to 6 weeks. Early treatment is usually very complicated. Although EANO-ESMO clinical practice guidelines of leptomeninges of metastasis have been presented [2], the recommendations based on the evidence are mostly based on expert opinion with a low level of consensus. Given the limitations in the diagnosis and treatment of $\mathrm{MC}$, missed diagnoses and delayed treatment are inevitable. Therefore, it is urgent to find a potential and effective treatment for MC.

Circulating tumor DNA (ctDNA) consists of short, double stranded DNA fragments that are released by tumors. It has been shown that plasma ctDNA can be used to characterize and monitor tumors [3-6]. ctDNA is a quantitative measure, and the concentration at a given time-point appears to be generally proportional to tumor progression [6-8]. Generally, ctDNA can be measured

(c) The Author(s). 2019 Open Access This article is distributed under the terms of the Creative Commons Attribution 4.0 International License (http://creativecommons.org/licenses/by/4.0/), which permits unrestricted use, distribution, and 
from different noninvasive sources, such as blood plasma, sputum, urine, or cerebrospinal fluid (CSF). Therefore, this enables convenient testing and real time monitoring of disease dynamics. There are various applications of ctDNA analysis, including therapy response monitoring, identification of resistance mechanisms, and relapse prediction. It has been noted, that next-generation sequencing (NGS) based ctDNA analysis has been able to identify epidermal growth factor receptor $(E G F R)$ mutations in plasma with high specificity and sensitivity.

CSF, which can have intimate contact with tumor cells, is an easily obtained body fluid that reflects the underlying pathological state of the central nervous system. Recently, ctDNA has been found in the CSF of patients with brain tumors $[9,10]$. Additionally, a case report underscored the potential of CSF-derived ctDNA analysis in the management of patients with human epidermal growth factor receptor-positive breast cancer [11]. It has been found that CSF ctDNA levels longitudinally fluctuate and follow changes in brain tumor burden variations, which provides a biomarker for monitoring brain malignancies [12]. Therefore, CSF ctDNA levels can follow tumor development, which is more sensitive and informative than traditional imaging. Moreover, it is more representative of brain tumor genomic alterations than plasma, and it can identify gene mutations such as EGFR [12].

In this study, we used a highly sensitive and specific method to analyze EGFR mutations in ctDNA with NGS technology. Here, we present a case of a MC patient, who was provided treatment based on analysis of EGFR mutations in ctDNA of their CSF.

\section{Case presentation}

According to the kit instructions, cell free DNA (cfDNA) and genomic DNA (gDNA) were extracted by the QIAamp Circulating Nucleic Acid kit (QIAGEN) Circulating Nucleic Acid Kit extraction kit and blood genome DNA extraction kit, respectively. The CSF samples were taken from $\mathrm{a}-80^{\circ} \mathrm{C}$ refrigerator and thawed at room temperature before extraction $(8 \mathrm{ml} \mathrm{CSF}$ and $200 \mu \mathrm{l}$ cell suspension). It was necessary to control the pressure of the vacuum pump (the calibration of the vacuum pump was first set to maximum and then slowly set to 0 ) to ensure that the CSF samples were integrated with the adsorption column. Before the sample library was constructed, the concentration and size of cfDNA fragments were quantitatively detected by Qubit 3 and $2100-\mathrm{H}$. The KAPA Hyper Prep Kit was used to construct the precapture (terminal repair, A-ESRiT connector $(15 \mu \mathrm{M})$, connection and PCR amplification). The capture of the target area was based on chip hybridization and liquid hybridization capture products from the SureSelect
QXT rapid hybridization kit (Agilent Company). A total of 120 cancer-associated genes were included in the gene panel, which was used for target region capture. The library was then tested by Agilent 2100 and Q-PCR (including size detection of the insertion fragment and concentration calculation of the effective fragment). Lastly, the Illumina NextSeq 500 system was used for sequencing.

After trimming the adapter sequences, and filtering and removing poor quality reads using flexbar software (version 2.7.0, https://sourceforge.net/projects/flexbar/), the clean fastq (the raw data file format of from sequencing) data were generated from the raw data from the NEXT SEQ 500 runs. Then, the clean fastq data was aligned to hg19 (GRCH37) and assembled through BWA-sampe (Burrows Wheeler Aligner software version 0.7.12-r1039, https://sourceforge.net/projects/bio-bwa/ files/). The polymerase chain reaction (PCR) duplicates were removed by MarkDuplicates in Picard Tools (version 1.124, http://broadinstitute.github.io/picard/). Variant calling was performed in the CSF (CerebroSpinal Fluid) by GATK (GenomeAnalysisTK version 3.6, https://software.broadinstitute.org/gatk/). All variants were annotated by ANNOVAR (version 20,160,201, http://annovar.openbioinformatics.org/en/latest/). Lastly, variation frequency $(>0.5 \%)$ was used to eliminate erroneous base calling and generate the final mutations. Manual verification was performed by IGV (Integrative Genomics Viewer version 2.3.72, http://software.broad institute.org/software/igv/).

Finally, a 19-Del mutation in the exon of the EGFR gene was found in gene detection of a patient's pleural effusion on March 11, 2015. He took $250 \mathrm{mg}$ oral gefitinib, once a day, for 11 months beginning in December 2015. On the 3rd of November 2016, he came to the hospital with dizziness, headache and blurred vision that was transient. He began to take $4 \mathrm{mg}$ oral zoledronic acid, once a month, to prevent bone metastasis. He came to the department of neurology when his neurological symptoms appeared. Pulmonary CT results showed that his left lung appeared as a placeholder (Fig. 1a). A cerebrospinal cytology exam was taken after admission. Several tumor cells could be seen in the cerebrospinal fluid (Fig. 1b). The immunohistochemistry showed positive staining for NapsinA and TTF1 (Fig. 2). The cerebrospinal cytology exam showed that the patient had meningeal carcinomatosis. After that, a lumbar puncture and an intrathecal injection of dexamethasone $(5 \mathrm{mg})$ and methotrexate $(10 \mathrm{mg})$ were conducted twice a week to control meningeal metastases. During this process, the patient was in stable condition and there appeared to be no significant disease progression or side effects of the medicine. At this same time, an appropriate amount of cerebrospinal fluid was preserved for next 

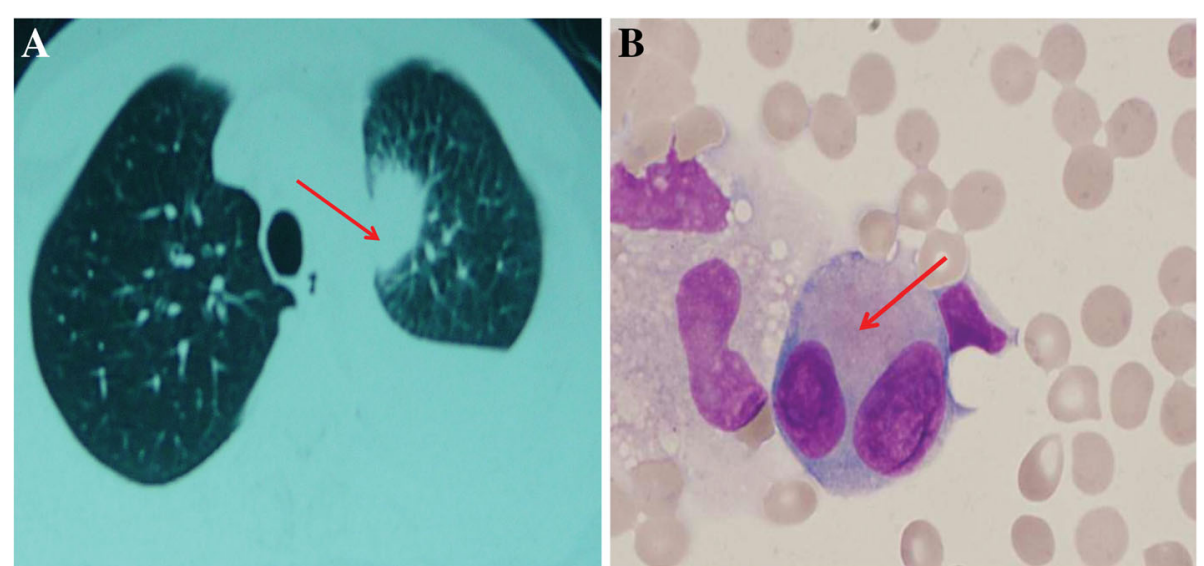

Fig. 1 Pulmonary Magnetic Resonance imaging and CSF cytology exams, (Magnification: 1000X) before adjusting the medication based on the NGS. a Left lung placeholder before hospital admission. The red arrow identifies the placeholder. b Cell types in CSF. CSF showed several tumor cells. Lymphocyte cells accounted for $20 \%$ of the cell populations, monocytes accounted for $22 \%$, activated monocytes accounted for $40 \%$, and neutrophils accounted for $18 \%$. The red arrow identifies the tumor cell

generation sequencing (NGS). A 19-Del mutation in the exon of the EGFR gene (mutation frequency: 30.8\%) and a 20-T790 M mutation in the exon of the EGFR gene (mutation rate $0.85 \%$ ) were found in the NGS. In November 2016, the patient discontinued gefitinib and began to take 90-100 mg of oral AZD9291 once a day. After adjusting the medication based on the results of the NGS, the headache of the patient was noticeably reduced, and his condition gradually stabilized. In February 2017, a pulmonary CT showed that the lesion in the superior lobe of the left lung was significantly reduced (Fig. 3a). At this same time, a cerebrospinal cytology exam showed that there were no tumor cells in his cerebrospinal fluid (Fig. 3b). The KPS score increased to 90 from 80 (before treatment), and the ECOG score was still 3.

\section{Discussion and conclusions}

Precision medicine, which relies on genomic information from the tumor, has been applied to cancer treatment [13]. The difficulty of acquiring tumor tissue and the inability to detect ctDNA in plasma leads to a particular challenge for central nervous system tumors [6]. In cancer patients, CSF is the best source of tumor DNA. Presently, some scholars are engaged in related research of tumor DNA in CSF $[12,14]$. The development of ctDNA detection has been limited, as there is low detection sensitivity in first generation sequencing (represented by Sanger sequencing). NGS technology has high sensitivity and specificity to distinguish between similar sequences, which makes ctDNA detection possible [15]. However, it is not clear whether a routine lumbar puncture and

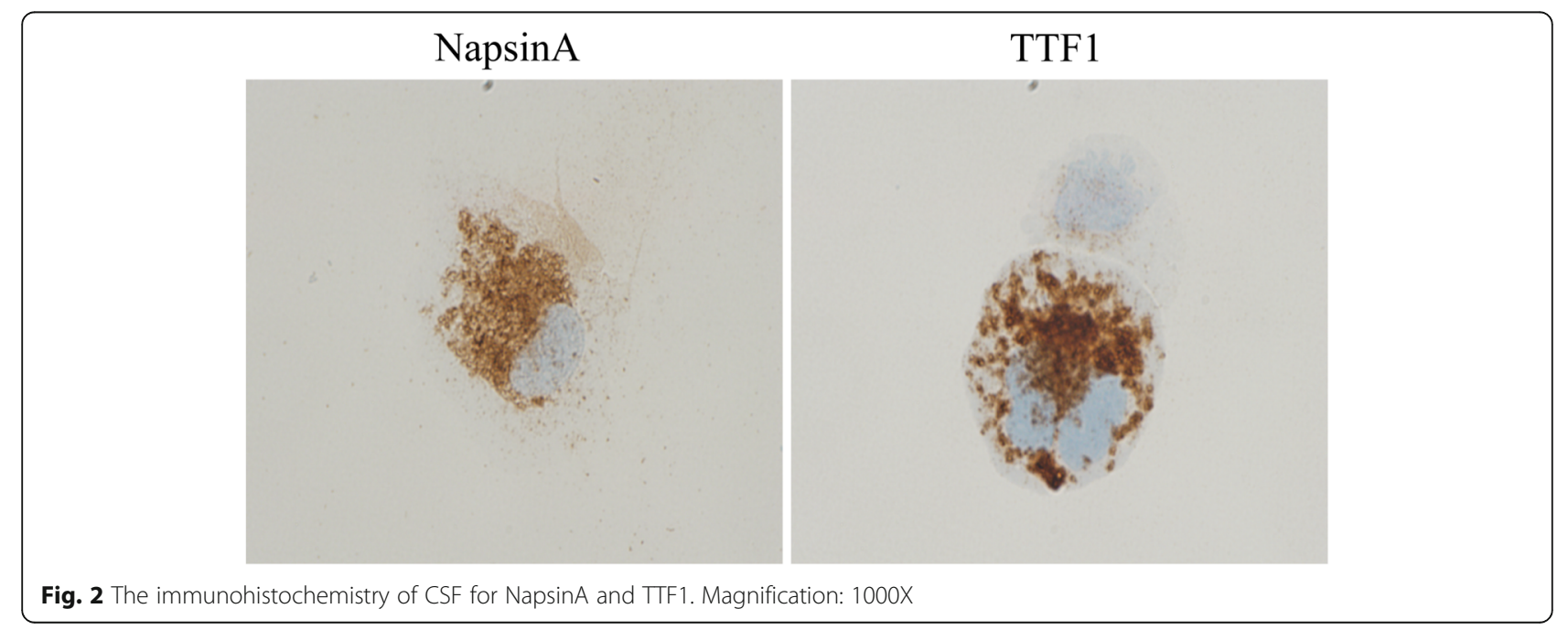



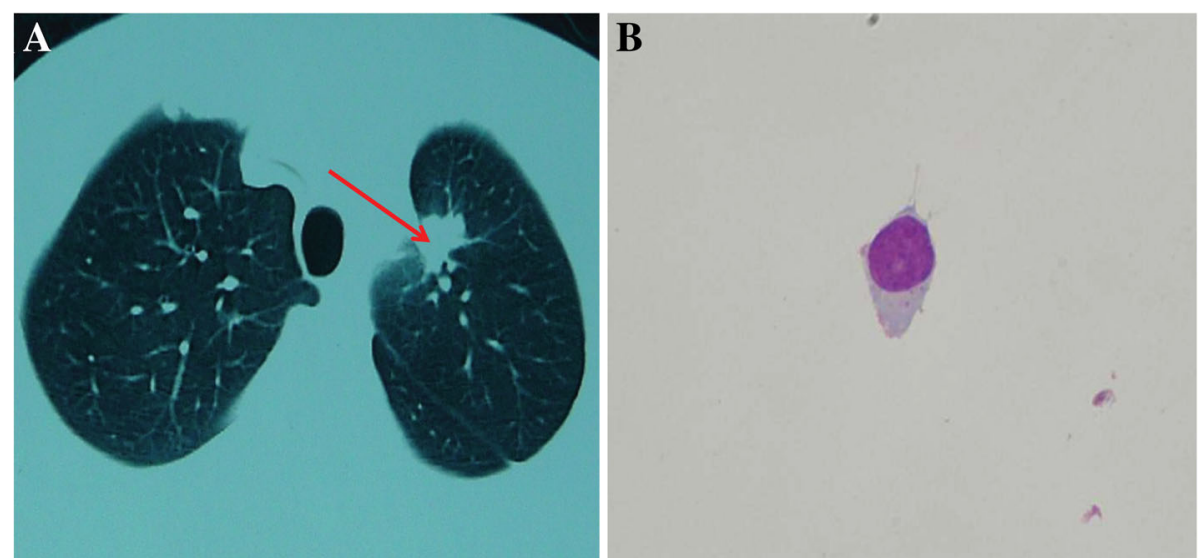

Fig. 3 Pulmonary Magnetic Resonance imaging and CSF cytology exams, (Magnification: 1000X) after adjusting the medication based on the NGS. a Lesion in the superior lobe of the left lung was significantly reduced after adjusting the targeted drug therapy. The red arrow identifies the placeholder. Figure 2 CSF cytology exams. Magnification: 1000X. b Cell types in CSF. CSF showed 17 lymphocytes, 3 monocytes, 2 activated monocytes and 2 neutrophilic granulocytes

targeted NGS can be used to identify tumor-associated DNA and provide valuable clinical guidance for treatment response.

It is an important potential application of CSF gene analysis to determine drug resistance mechanisms in patients. Through NGS, we detected ctDNA in the CSF of one MC patient with primary lung cancer, and conducted targeted therapy based on these results. Targeted gene therapy was effective for the primary tumor, however, tumor development of the central nervous system continued to progress. The patient appeared with symptoms in his head after taking oral gefitinib for 11 months. We took CSF from the patient diagnosed with MC to use for NGS. We found a 20-T790 M mutation in the exon of the EGFR gene, which is the most common factor seen in resistance to a generation of EGFR tyrosine kinase inhibitors (TKIs) in nonsmall cell lung cancer [16]. The mutation rate was $0.85 \%$, which suggests that the patient had resistance to gefitinib. Recently, there have been several EGFR TKIs approved, such as, first-generation gefitinib and erlotinib, second-generation afatinib, and third generation osimertinib. AZD9291 is an irreversible third generation EGFR TKI, which showed a high response rate in patients whose tumors harbored EGFR-T790 M and the capability to penetrate the blood-brain barrier $[17,18]$. The condition of the patient improved after oral administration of AZD9291, which suggests the significance of NGS of CSF ctDNA in disease assessment and medication guidance. However, it is worth mentioning that a 19 deletion mutation in the exon of the EGFR gene is still present, which suggests that targeted medicines possess a certain sensitivity. After taking oral AZD9291, the patient's clinical symptoms improved.

In conclusion, our study confirmed that ctDNA can be detected in the CSF from MC patients. In addition, we used NGS technology and found a 20-T790 M in the exon of the EGFR gene in ctDNA from CSF, which may be helpful in providing drug treatment guidance.

\section{Abbreviations}

cfDNA: cell free DNA; CSF: Cerebrospinal fluid; ctDNA: circulating tumor DNA: EGFR: Epidermal growth factor receptor; gDNA: genomic DNA; MC: Meningeal carcinomatosis; NGS: Next generation sequencing; PCR: Polymerase chain reaction; TKIs: Tyrosine kinase inhibitors

\section{Acknowledgements}

Not applicable.

\section{Funding}

Not applicable.

\section{Availability of data and materials}

The dataset supporting the conclusions of this article is included within the article.

\section{Authors' contributions}

$J C, Y Z, W H, Y I Z, R G, Q L$ and $X L$ analyzed the detailed information about the patient and expounded the diagnostic method in this case report. XG was a major contributor in writing the manuscript. JH and HB helped draft the manuscript and performed a literature search. All authors have read and approved the manuscript and ensure that this is the case.

\section{Ethics approval and consent to participate}

This study was approved by the ethical committee of the Second Hospital of Hebei Medical University. The written informed consent was signed and obtained from each patient before use of their personal information for research purposes.

\section{Consent for publication}

The subjects gave written informed consent for the publication of any associated data and accompanying images.

\section{Competing interests}

The authors declare that they have no competing interests.

\section{Publisher's Note}

Springer Nature remains neutral with regard to jurisdictional claims in published maps and institutional affiliations. 
Received: 26 July 2018 Accepted: 4 March 2019

Published online: 09 March 2019

\section{References}

1. Grossman SA, Krabak MJ. Leptomeningeal carcinomatosis. Cancer Treat Rev. 1999:25(2):103-19.

2. Le RE, Weller M, Brandsma D, Van dBM, De AE, Henriksson R, Boulanger T, Peters S, Watts C, Wick W. EANO-ESMO clinical practice guidelines for diagnosis, treatment and follow-up of patients with leptomeningeal metastasis from solid tumours. Ann Oncol. 2017;28(suppl_4):iv84.

3. Murtaza M, Dawson SJ, Tsui DW, Gale D, Forshew T, Piskorz AM, Parkinson C, Chin SF, Kingsbury Z, Wong AS, et al. Non-invasive analysis of acquired resistance to cancer therapy by sequencing of plasma DNA. Nature. 2013; 497(7447):108-12.

4. Dawson SJ, Tsui DW, Murtaza M, Biggs H, Rueda OM, Chin SF, Dunning MJ, Gale D, Forshew T, Mahler-Araujo B, et al. Analysis of circulating tumor DNA to monitor metastatic breast cancer. N Engl J Med. 2013;368(13):1199-209.

5. De Mattos-Arruda L, Weigelt B, Cortes J, Won HH, Ng CK, Nuciforo P, Bidard FC, Aura C, Saura C, Peg V, et al. Capturing intra-tumor genetic heterogeneity by de novo mutation profiling of circulating cell-free tumor DNA: a proof-of-principle. Ann Oncol. 2014;25(9):1729-35.

6. Bettegowda C, Sausen M, Leary RJ, Kinde I, Wang Y, Agrawal N, Bartlett BR, Wang H, Luber B, Alani RM, et al. Detection of circulating tumor DNA in early- and late-stage human malignancies. Sci Transl Med. 2014;6(224): $224 \mathrm{ra} 224$.

7. Diehl F, Schmidt K, Choti MA, Romans K, Goodman S, Li M, Thornton K, Agrawal N, Sokoll L, Szabo SA, et al. Circulating mutant DNA to assess tumor dynamics. Nat Med. 2008;14(9):985-90.

8. Jung K, Fleischhacker M, Rabien A. Cell-free DNA in the blood as a solid tumor biomarker--a critical appraisal of the literature. Clin Chim Acta. 2010 411(21-22):1611-24.

9. Segal MB. Extracellular and cerebrospinal fluids. J Inherit Metab Dis. 1993; 16(4):617-38.

10. Pan W, Gu W, Nagpal S, Gephart MH, Quake SR. Brain tumor mutations detected in cerebral spinal fluid. Clin Chem. 2015;61(3):514-22.

11. Siravegna G, Geuna E, Mussolin B, Crisafulli G, Bartolini A, Galizia D, Casorzo L, Sarotto I, Scaltriti M, Sapino A, et al. Genotyping tumour DNA in cerebrospinal fluid and plasma of a HER2-positive breast cancer patient with brain metastases. ESMO Open. 2017;2(4):e000253.

12. De Mattos-Arruda L, Mayor R, Ng CK, Weigelt B, Martinez-Ricarte F, Torrejon D, Oliveira M, Arias A, Raventos C, Tang J, et al. Cerebrospinal fluid-derived circulating tumour DNA better represents the genomic alterations of brain tumours than plasma. Nat Commun. 2015;6:8839.

13. Pentsova El, Shah RH, Tang J, Boire A, You D, Briggs S, Omuro A, Lin X Fleisher M, Grommes C, et al. Evaluating Cancer of the central nervous system through next-generation sequencing of cerebrospinal fluid. J Clin Oncol. 2016;34(20):2404-15

14. Wang Y, Springer S, Zhang M, McMahon KW, Kinde I, Dobbyn L, Ptak J, Brem H, Chaichana K, Gallia GL, et al. Detection of tumor-derived DNA in cerebrospinal fluid of patients with primary tumors of the brain and spinal cord. Proc Natl Acad Sci U S A. 2015;112(31):9704-9.

15. Fox EJ, Reid-Bayliss KS, Emond MJ, Loeb LA. Accuracy of next generation sequencing platforms. Next Gener Seq Appl. 2014;1. https://doi.org/10.4172/ jngsa.1000106.

16. Yu HA, Arcila ME, Rekhtman N, Sima CS, Zakowski MF, Pao W, Kris MG, Miller VA, Ladanyi M, Riely GJ. Analysis of tumor specimens at the time of acquired resistance to EGFR-TKI therapy in 155 patients with EGFR-mutant lung cancers. Clin Cancer Res. 2013;19(8):2240-7.

17. Cross DA, Ashton SE, Ghiorghiu S, Eberlein C, Nebhan CA, Spitzler PJ, Orme JP, Finlay MR, Ward RA, Mellor MJ, et al. AZD9291, an irreversible EGFR TKI, overcomes T790M-mediated resistance to EGFR inhibitors in lung cancer. Cancer Discov. 2014;4(9):1046-61.

18. Steuer CE, Khuri FR, Ramalingam SS. The next generation of epidermal growth factor receptor tyrosine kinase inhibitors in the treatment of lung cancer. Cancer. 2015;121(8):E1-6.

\section{Ready to submit your research? Choose BMC and benefit from:}

- fast, convenient online submission

- thorough peer review by experienced researchers in your field

- rapid publication on acceptance

- support for research data, including large and complex data types

- gold Open Access which fosters wider collaboration and increased citations

- maximum visibility for your research: over $100 \mathrm{M}$ website views per year

At $\mathrm{BMC}$, research is always in progress.

Learn more biomedcentral.com/submissions 